\title{
Impact of Hydrogen Gas on the Inverse Spin Hall Effect in Palladium- Cobalt Bi-Layer Films
}

\author{
S. Watt ${ }^{1}$, R. Cong ${ }^{1,2}$, C. Lueng ${ }^{1}$, M. Sushruth1, P.J. Metaxas ${ }^{1}$, and M. Kostylev ${ }^{1}$ \\ ${ }^{1}$ School of Physics and Astrophysics, University of Western Australia, Crawley 6009, WA, Australia \\ ${ }^{2}$ University of Science and Technology of China, Hefei, China
}

Received 1 Apr 2016, revised 15 Apr 2016, accepted 20 Apr 2016, published 1 Jun 2016, current version 15 Jun 2016. (Dates will be inserted by IEEE; "published" is the date the accepted preprint is posted on IEEE Xplore ${ }^{\circledR}$; "current version" is the date the typeset version is posted on Xplore ${ }^{\circledR}$.

\begin{abstract}
The influence of hydrogen gas absorption on the inverse Spin Hall Effect (iSHE) in the material is measured in $\mathrm{Co} / \mathrm{Pd}$ bi-layer films. The iSHE is driven by ferromagnetic resonance in the cobalt layer and manifests as a dc voltage across the Pd layer. In the presence of hydrogen gas, the iSHE peak shifts downwards in applied field together with the ferromagnetic resonance absorption peak for the material. In parallel, an increase in the iSHE peak height and width is observed.
\end{abstract}

Index Terms—-Magnetic and spintronic materials, Hall effect, magnetic sensors, magnetization dynamics, spin currents.

\section{INTRODUCTION}

Palladium (Pd)/cobalt (Co) bi-layer films demonstrate a host of important magnetic and spintronic effects. Firstly, a strong perpendicular magnetic anisotropy (PMA) is induced at the interface between the two layers [Draaisma 1987]. Also these films are characterized by a strong Spin Hall Effect. The inverse Spin Hall voltage across the $\mathrm{Pd}$ layer can be observed during driven ferromagnetic resonance (FMR) in the Co layer [Ando 2010] during which a strong spin-pumping effect [Shaw 2012] across the interface leads to a strong inverse Spin Hall Effect (iSHE) in the Pd layer.

Unique for palladium is its strong affinity to hydrogen gas $\left(\mathrm{H}_{2}\right)$ at room temperature and normal atmospheric pressure. Furthermore, it has been demonstrated that absorption of hydrogen gas affects the effective field of interface PMA [Lederman 2004, Munbodh 2012, Lin 2013]. This results in a downward shift (in field) of the in-plane FMR peak for the cobalt layer [Chang 2013]. The shift is consistent with a hydrogen-induced decrease in PMA.

It is possible to exploit the hydrogen-gas induced FMR peak shift to measure hydrogen gas concentration in the environment [Lueng 2016, Lueng 2017]. With the introduction of hydrogen fuel cells in the automotive industry, the demand for highly stable and sensitive hydrogen gas sensors has risen dramatically and there is an unsatisfied requirement for safer, more sensitive detection methods [Buttner 2011]. Many approaches to developing hydrogen gas sensors make use of Palladium's affinity to hydrogen gas. In particular, sensors exploiting FMR-based probing of the interfacial PMA in Pd/Co bilayer films have an important advantage: demonstrated capability of hydrogen gas concentration measurements in a very wide concentration range, including near $100 \%$ hydrogen concentration [Lueng 2017] (a concentration regime where most alternative approaches fail).

In the present paper we explore the effect of hydrogen gas on the iSHE response of $\mathrm{Co} / \mathrm{Pd}$ films when their Co layers are driven to FMR.
We demonstrate that the peak in theiSHE voltage across the Pd layer shifts downwards in applied field in the presence of the gas. This demonstrates the potential for FMR-based hydrogen gas sensing using a device-generated $d c$ output, here the iSHE voltage. In contrast, in our previous studies, the output signal of the magnetic gas sensor was a microwave voltage (e.g. [Lueng 2016]). Using the iSHE eliminates the need to convert the originally microwave-frequency output of the sensor into a low-frequency signal that is suited to detection with simple electronics. We also note that the observed $\mathrm{H}_{2-}$ induced shift of the iSHE peak is accompanied by a decrease in the peak linewidth and an increase in its height.

\section{EXPERIMENT}

Palladium/cobalt bilayer films were deposited onto silicon substrates using DC magnetron sputtering at an argon working pressure of $8 \mathrm{mTorr}$. The system base pressure prior to sputtering was on the order of $10^{-7}$ Torr. Each sample consists of a $10 \mathrm{~nm}$ layer of cobalt with a capping palladium layer of varying thickness $(4.4 \mathrm{~nm}$ $15 \mathrm{~nm}$ ). A reference $10 \mathrm{~nm}$ thick single-layer cobalt film was also grown.

Leads were attached to opposite edges of each sample using a conductive epoxy. Similar to [Ando 2010], the samples were mounted on a polystyrene holder and placed in the center of a microwave cavity where they were subject to an in-plane external magnetic field (see Fig. 1). The advantage of the cavity method is that, theoretically, the microwave electric field in the middle of a $\mathrm{TE}_{102}$ cavity is precisely zero. This implies that the effects of spin rectification, anisotropic magneto-resistance, and spin-torque rectification [Harder 2016], which like the iSHE can appear as voltages across the sample, are vanishing for a proper orientation of the applied magnetic field with respect to the sample. However, the metallic sample grown on a thick dielectric substrate with dielectric permittivity $\varepsilon=11$ represents a system with broken symmetry. Therefore its insertion into the cavity 
may lead to a slight break in symmetry of the microwave field, potentially resulting in a small contribution from these effects to the total DC voltage. To check the actual strength of this contribution, measurements were carried out with the reference sample, as detailed below.

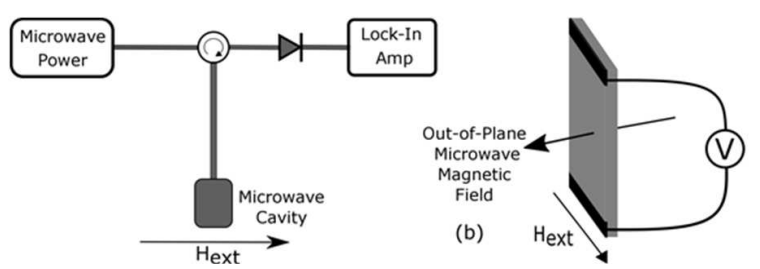

Fig. 1. (a) Microwave cavity FMR configuration. (b) Voltage generated across the sample is measured perpendicular to the external static magnetic field and incident microwave field via leads attached with conductive epoxy.

The microwave power source was tuned to the cavity resonance at approximately $9.5 \mathrm{GHz}$ and the external field swept to induce FMR in the cobalt layer. The iSHE-induced voltage across the sample length perpendicular to the in-plane field was measured using a nanovoltmeter.

With the same equipment, we also measure conventional fieldresolved FMR spectra. The Varian-IV® microwave cavity has builtin modulation coils which are used to generate a small ac. modulating field at $20.1 \mathrm{kHz}$ which is parallel to the static magnetic field. The spectra are obtained by measuring the microwave signal reflected from the cavity using a microwave diode. To do this, the rectified voltage from the diode's output port is fed into a lock-in amplifier locked to the frequency of the modulation field ("Field Modulated FMR"). Accordingly, the digital output of the lock-in scales as the first derivative of the resonance absorption signal with respect to the applied field (“differential FMR trace”).

Measurements carried out on the reference single-layer sample revealed a strong FMR absorption signal but there was no measurable dc voltage across the sample. This confirmed that spin rectification and anisotropic magneto-resistance are negligible in our geometry.

Each $\mathrm{Pd} / \mathrm{Co}$ sample is measured first in nitrogen gas atmosphere and then exposed to a hydrogen gas containing atmosphere. Typical FMR and iSHE responses are shown in Fig. 2. We use 3\% hydrogen gas diluted in $97 \%$ of nitrogen gas. Note that the flammability threshold for $\mathrm{H}_{2}$ in air is $4 \%$ and an air- $\mathrm{H}_{2}$ mix becomes explosive above $18 \%$ of hydrogen [Hubert 2011, Greco 2012]. As the cavity together with its feeding waveguide represents a large vessel filled with a potentially explosive gas, measurements were taken with the diluted $\mathrm{H}_{2}$ only to ensure safety of the experiment. It is straightforward to extrapolate the results we obtain in the present work onto higher $\mathrm{H}_{2}$ concentrations based on the results from [LuengLupo 2016, Lueng 2017] of FMR characterization of similar samples in a broad range of $\mathrm{H}_{2}$ concentrations.

\section{RESULTS}

Upon exposure of the sample to the hydrogen containing gas mixture (referred to as " $3 \% \mathrm{H}_{2}$ " in the following), a distinct shift to lower fields of both iSHE and FMR peaks is evident. The iSHE peak is fitted with a complex-valued combination of Lorentzian and anti-
Lorentzian line-shapes (see e.g. $(5,6)$ in [Soh 2014]) and the FMR peak with a complex-valued combination of the first derivatives of those shapes. From these fits, the resonance position and linewidth are extracted.

The resonance fields for the samples in the virgin state and their change in the presence of $\mathrm{H}_{2}$ are shown in Fig. 3. One sees that the minimum resonance field corresponds to a sample with a $7.5 \mathrm{~nm}$ thick cap (Fig. 3(a)). The smallest FMR field corresponds to the largest effective PMA field. Interestingly, this sample shows the smallest FMR and iSHE peak shift in the presence of hydrogen gas (Fig3.(b)). We speculate that this may be related to a change in the crystal structure of the Pd layer as a function of its thickness, similar to that previously observed for heavy metals such as hafnium and tungsten [Liu 2015]. The fits also demonstrate a very good agreement between the FMR and iSHE peak positions for all the samples. This is consistent with the established understanding that the spin pumping effect by the precessing magnetization in the Co layer underlies the iSHE response (see e.g. theories in [Ando 2010, Soh 2014, Mosendz 2010].

The changes to the sample response upon hydrogenation are illustrated in Figs. 3 and 4. Firstly, we find a consistent decrease in the resonance field upon hydrogenation (Fig. 2 and 3(b)). The peak shift to lower fields is consistent with previous studies [Chang 2013, Lueng-Lupo 2016, Lueng 2013, Lueng 2015, Lueng 2017] and is related to a decrease in the effective field of the interface perpendicular magnetic anisotropy. A clear evidence for this origin of the shift is its scaling as $1 / L$ [Lueng 2015], where $L$ is the cobalt layer thickness. This type of scaling is characteristic to an interface effect which is PMA in the case of $\mathrm{Pd} / \mathrm{Co}$ bi-layer films.

The hydrogen gas induced shift of the iSHE peak is the main finding of this work. It demonstrates the possibility of reading out the state of a resonance-based magnetic hydrogen gas sensor directly at dc using the iSHE.
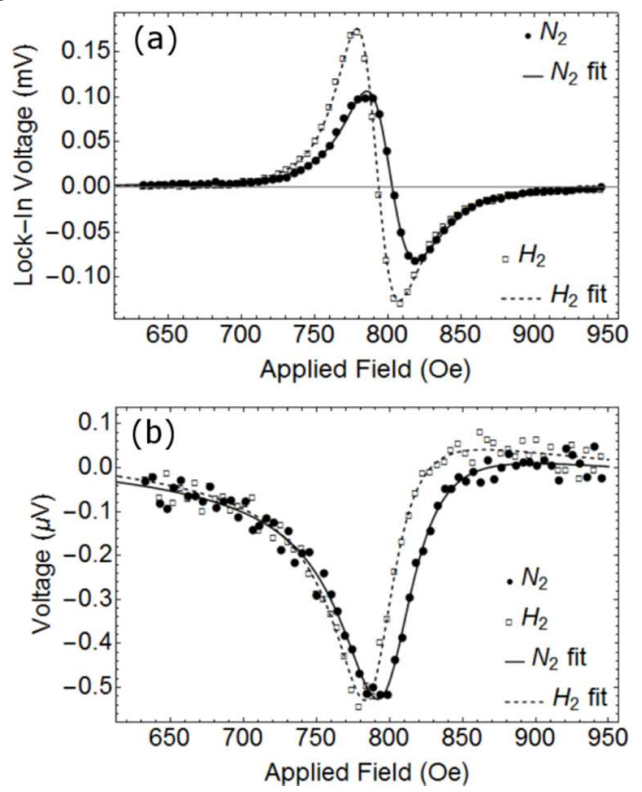

Fig. 2. (a) FMR signal and fit for $\mathrm{Co}(10 \mathrm{~nm}) / \mathrm{Pd}(6.3 \mathrm{~nm})$ sample in nitrogen (circles/solid line) and $3 \% \quad \mathrm{H} 2$ (squares/dashed line) atmospheres. (b) iSHE voltage and Lorentzian fit measured across $\mathrm{Co}(10 \mathrm{~nm}) / \mathrm{Pd}(6.3 \mathrm{~nm})$ sample in nitrogen (circles/solid line) and $3 \% \mathrm{H} 2$ (squares/dashed line) atmospheres. 

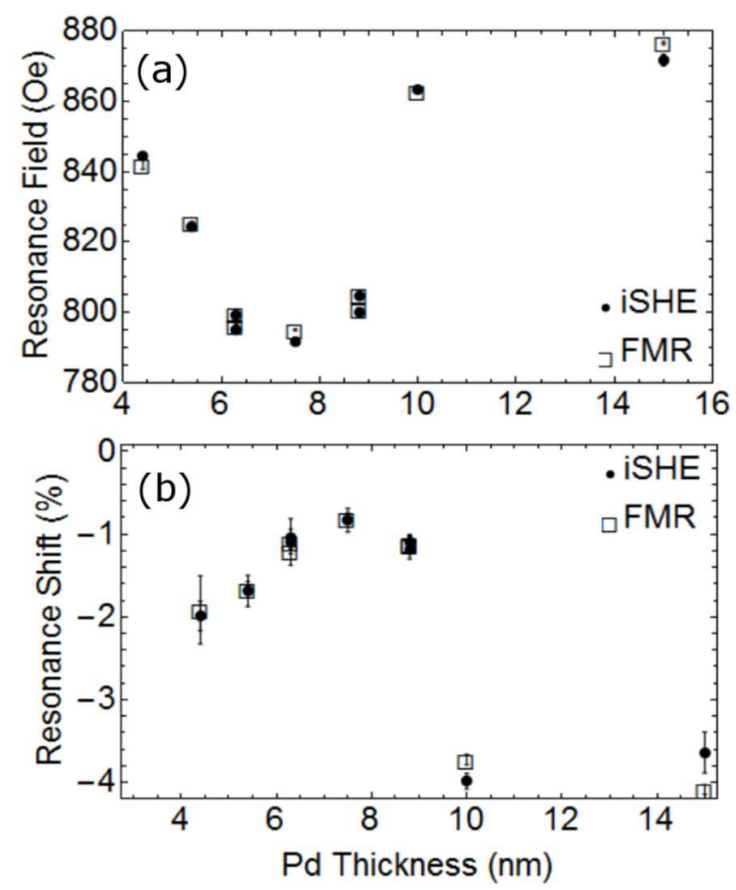

Fig. 3. (a) FMR peak positions for the samples in their virgin state (i.e. in a $\mathrm{N}_{2}$ atmosphere). (b) Percentage shift in resonance position upon hydrogenation. Squares: measured via FMR; circles: measured via iSHE.

We also find that absorption of $\mathrm{H}_{2}$ induces a decrease in the linewidth (Fig. 4(b)) of both FMR and iSHE peaks. This observation is consistent with our previous studies in which we also saw a decrease in the linewidth of the FMR peak [Chang 2013, LuengMetaxas 2016]. We do however observe a difference in the linewidths measured through the iSHE and FMR channels (Fig. 4(a)) which exceeds error margins of the fit procedure. Analyzing this difference is beyond the scope of the present paper whose main focus is on the changes in the iSHE signal in the presence of $\mathrm{H}_{2}$.

These changes are clearly visible in Fig. 2, 3(b) and 4(b). Also, from comparison of Fig. 4(b) and 3(b) one sees that the decrease in the linewidth correlates with the change in the peak position - the smallest change in the linewidth is observed at the center of the Pd thickness range where the peak shift is the smallest.

In our previous work, we consistently observed an increase in the height of the FMR peak upon hydrogen gas absorption [Chang 2012, Lueng-Lupo 2016, Lueng-Metaxas 2016, Lueng 2017]. The same trend is seen in Fig. 2 (a). However, from Fig. 2 (b) one notices that the change in the height of the iSHE peaks is smaller. This tendency was observed for all measured samples. Fig. 4 (c) illustrates this effect - it shows the ratio of resonance peak heights for the FMR and ISHE traces in $3 \% \mathrm{H}_{2}$ and $\mathrm{N}_{2}$ atmospheres.

Similar to our previous studies, the change in the height of the FMR peaks is larger than expected from the linewidth reduction plus a contribution from the change in coupling of dynamic magnetization to the driving microwave field due to a change in PMA. Hence, we conclude that there is one more mechanism which alters the coupling of dynamic magnetization to the driving field in the presence of hydrogen gas. We suppose that the mechanism only affects the Pd layer and the interface, since Co does not absorb hydrogen. One possible mechanism could be a modified spin pumping. However, this would contribute to the observed decrease in the linewidth and modify the peak amplitude proportionally. As we find a contribution to the FMR amplitude change which does not correlate with the linewidth change, there should be one more mechanism for the amplitude growth which has yet to be found. Results of a more detailed investigation of this effect will be published elsewhere [Schefer 2017].
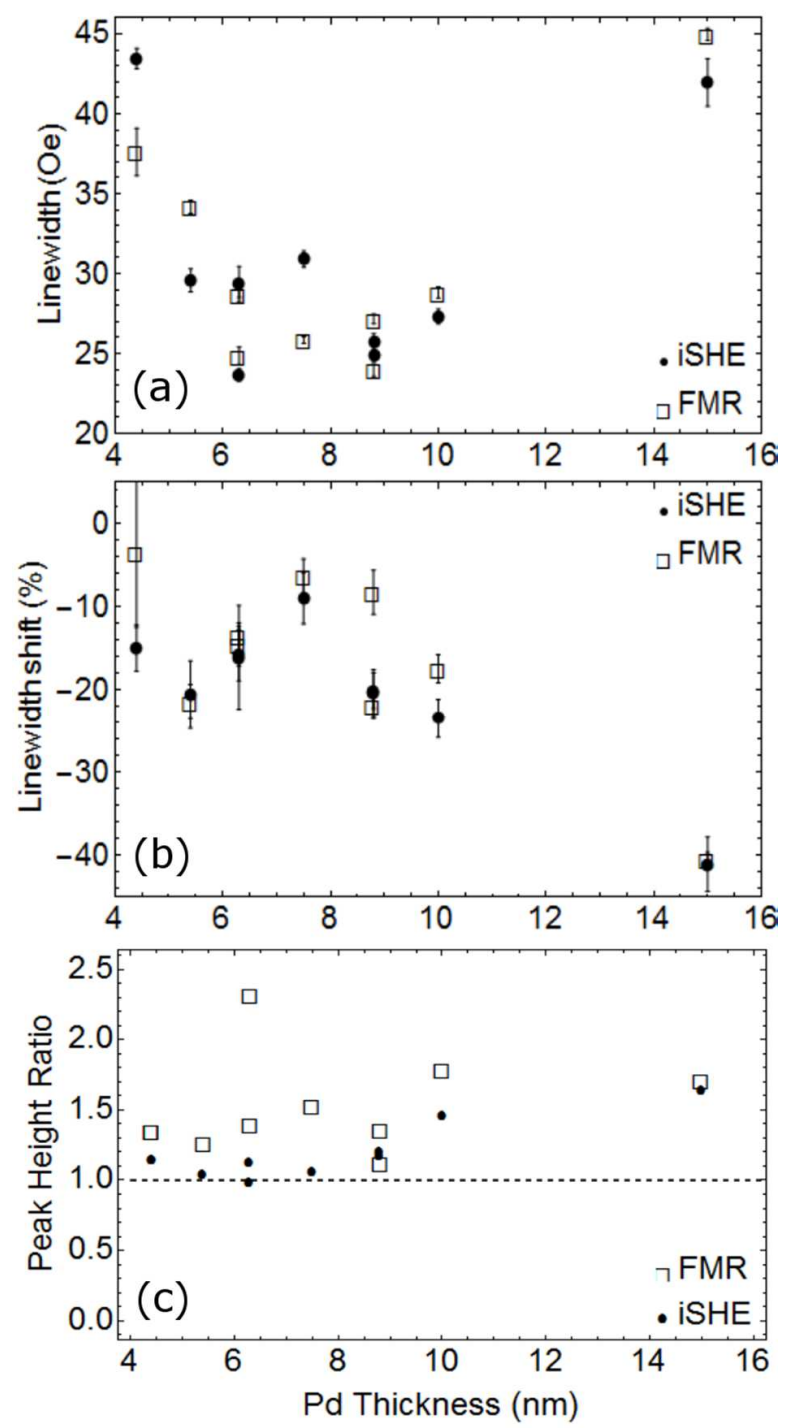

Fig. 4. (a) Resonance linewidth for the samples in their virgin state (il.e. in a N2) atmosphere). (b) Change in FMR and iSHE linewidths upon hydrogenation. (c) Ratio of peak heights between nitrogen and $3 \% \mathrm{H} 2$ atmospheres. Squares: measured via FMR; circles: measured via iSHE.

To understand the difference between the changes to the heights of the iSHE and FMR peaks we attempt to quantify the changes to the iSHE response using a method similar to that employed in [Ando 2010] where the same cavity FMR approach was used to measure the iSHE response of $\mathrm{Pd} / \mathrm{Co}$ films. Our analysis suggests that one possible reason for the decrease in the iSHE peak amplitude is a decrease in the Spin Hall Angle in the presence of $\mathrm{H}_{2}$. However, the absolute value of the decrease in the angle could not be extracted, because the 
registered change in the iSHE amplitude due to absorption of hydrogen is comparable with a numerical error which accumulates during the multi-step calculation of the Spin Hall Angle based on the raw FMR and iSHE data.

\section{CONCLUSION}

In conclusion, in this work we carried out investigation of the influence of hydrogen gas absorption by the Pd layer of bi-layered films containing Pd and Co layers on the inverse Spin Hall Effect in the material, the latter driven by ferromagnetic resonance in the cobalt layer. We found that in the presence of hydrogen gas the iSHE peak shifts downwards in the applied field together with the ferromagnetic resonance absorption peak for the material. In parallel, an increase in the iSHE peak height and a decrease in the peak width were observed for most of the samples. The iSHE peak shift magnitude is the same as for the FMR absorption peak and the peak linewidths are close. This implies that the iSHE response sensitivity to hydrogen gas concentration is the same as for the FMR response. The latter was investigated in detail in [Lueng-Lupo 2016, Lueng 2017].

This finding opens up a pathway to design a magnetic-film based hydrogen gas sensor whose output signal will be a dc voltage. This represents an advantage with respect to previously suggested magnetic hydrogen gas sensor concepts based on a microwave frequency sensor output [Lueng-Lupo 2016].

\section{ACKNOWLEDGMENT}

The authors acknowledge the facilities, and the scientific and technical assistance of the Australian National Fabrication Facility at the Centre for Microscopy, Characterisation \& Analysis, The University of Western Australia, a facility funded by the University, State and Commonwealth Governments. M.K. acknowledges his Senior Research Award from the University of Western Australia. P.J.M. acknowledges support from the Australian Research Council's Discovery Early Career Researcher Award Scheme (DE120100155).

\section{REFERENCES}

H.J.G. Draaisma, W.J.M. de Jonge, and F.J.A. den Broeder, J.Mag.Mag.Mat. 66, 351 (1987).

K. Ando and E. Saitoh, J.Appl.Phys. 108, 113925 (2010)

J.M. Shaw, H.T. Nembach and T.J. Silva, Phys.Rev.B 85054412 (2012).

D. Lederman, Y. Wang, E.H. Morales, R.J. Matelon, G.B. Cabrera, U.G. Volkmann and A.L. Cabrera, Appl.Phys.Lett. 85, 615 (2004).

K. Munbodh, F.A. Perez, C. Keenan, and D. Lederman, J.Appl.Phys. 111, 123919 (2012). W.-C.Lin, C.J. Tsai, B.Y. Wang, C.H. Kao, and W.F. Pong, Appl.Phys.Lett. 102, 252404 (2013).

C.S. Chang, M. Kostylev, and E. Ivanov, Appl.Phys.Lett. 102, 142405 (2013).

C. Lueng, P. Lupo, P.J. Metaxas, M. Kostylev, and A.O. Adeyeye, Adv. Mat. Techn., 2016, 1600097 (2016).

C. Lueng, P.J. Metaxas, M. Sushruth, and M. Kostylev, Int.J.Hydr Ener. 423407 (2017).

W.J. Buttner, M.B. Post, R. Burgess, and C. Rivkin, Int.J.Hydrogen Energ. 36, 2462 (2011).

T.Hubert, L. Boon-Brett, G. Black, and U. Banach, Sen.Actuat. B 157, 329 (2011).

F. Greco, L. Ventrelli, P. Dario, B. Mazzolai, and V. Mattoli, Int. J. Hydr. Ener. 37, 1752 (2012).

J. Liu, T. Ohkubo, S. Mitani, K. Hono, and M. Hayashi, Appl. Phys. Lett. 107, 232408 (2015).

M. Harder, Y. Gui and C-M. Hu, Phys. Rep. 661, 1 (2016).

W.T. Soh, B. Peng, and C.K. Ong, J.Phys. D: Appl. Phys. 47, 285001 (2014).

O. Mosendz, J.E. Pearson, F.Y. Fradin, G.E. Bauer, S.D. Bader and A. Hoffmann, Phys.Rev.Lett. 104, 046601 (2010).

C. Lueng, P. Metaxas and M. Kostylev, COMMAD Conference Proceedings, Perth, WA, Australia, DOI: 10.1109/COMMAD.2014.7038643, 2015.

C. Lueng, P.J. Metaxas, and M. Kostylev, IEEE Trans Mag., 52, 4002503 (2016).
T. Schefer and M. Kostylev, "Effect of hydrogen gas on the FMR absorption amplitude of Pd/Co layered films", unpublished (2017). 